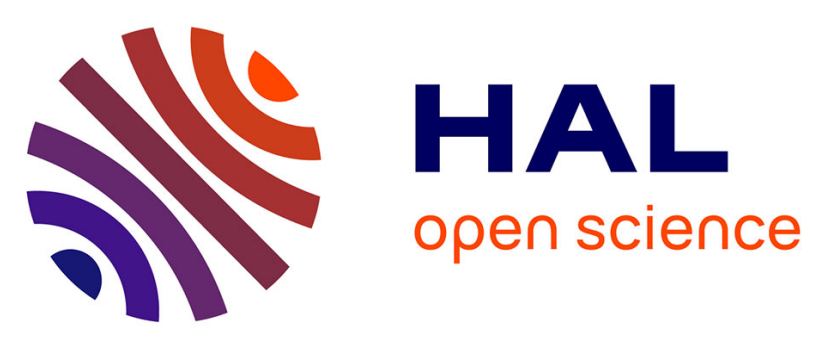

\title{
Work of Adhesion Measurements of MoS 2 Dry Lubricated 440C Stainless Steel Tribological Contacts
}

Simo Pajovic, Guillaume Colas, Aurélien Saulot, Mathieu Renouf, Tobin

Filleter

\section{- To cite this version:}

Simo Pajovic, Guillaume Colas, Aurélien Saulot, Mathieu Renouf, Tobin Filleter. Work of Adhesion Measurements of MoS 2 Dry Lubricated 440C Stainless Steel Tribological Contacts. Advanced Engineering Materials, 2017, 10.1002/adem.201700423 . hal-01598048

\section{HAL Id: hal-01598048 \\ https://hal.science/hal-01598048}

Submitted on 29 Sep 2017

HAL is a multi-disciplinary open access archive for the deposit and dissemination of scientific research documents, whether they are published or not. The documents may come from teaching and research institutions in France or abroad, or from public or private research centers.
L'archive ouverte pluridisciplinaire $\mathbf{H A L}$, est destinée au dépôt et à la diffusion de documents scientifiques de niveau recherche, publiés ou non, émanant des établissements d'enseignement et de recherche français ou étrangers, des laboratoires publics ou privés. 


\title{
Work of Adhesion Measurements of $\mathrm{MoS}_{2}$ Dry Lubricated 440C Stainless Steel Tribological Contacts
}

\author{
Simo Pajovic, Guillaume Colas, Aurélien Saulot, Mathieu Renouf, \\ and Tobin Filleter*
}

The tribological behavior of dry lubricants depends on their mechanical and physicochemical environment, making it difficult to predict in practice. Discrete Element Method-based modeling has been one successful approach to provide valuable insight into the tribology of dry lubricated contacts. However, it requires well-defined interactions between discrete elements, in particular between those simulating different materials. Measuring the properties governing those interactions, such as the work of adhesion (W), is therefore critical. The present work describes a method for measuring the $W$ between AISI440C steel and $\mathrm{MoS}_{2}$-based coatings used in spacecraft. Using Atomic Force Microscopy local asperity and adhesion measurements, the $W$ between steel microbeads and $\mathrm{MoS}_{2}$ coatings is determined at different stages in its wear life. The distributions of $W$ values in the worn coatings and pristine coatings agree well with earlier Time-of-Flight Secondary Ion Mass Spectroscopy studies on the physicochemistry of the samples, as well as contact angle measurements. Additional measurements between the same materials on a ball bearing from a real life-test unit of a spacecraft instrument also show a similar $W$ distribution, suggesting that the approach used here provides relevant data for use in numerical simulations.

\section{Introduction}

The tribological behavior of dry lubricants strongly depends on the surrounding physicochemical and mechanical environments, which makes it hard to predict. This is particularly true for space mechanisms whose lubrication must be sustained in the multiple environments, as they are successively tested on Earth and used in space. ${ }^{[1-5]}$ Moreover, tribological contacts are closed and

Prof. T. Filleter, S. Pajovic, Dr. G. Colas

Department of Mechanical and Industrial

Engineering, University of Toronto, Canada

E-mail: filleter@mie.utoronto.ca

Dr. A. Saulot

Université de Lyon, LaMCoS, INSA-Lyon, CNRS

UMR 5259, France

Dr. M. Renouf

Laboratoire de Mécanique et Génie Civil (LMCC),

CNRS, Université de Montpellier, Montpellier,

France impossible to observe without changing contact conditions by, for example, using transparent materials such as glass and sapphire. ${ }^{[6-9]}$ Therefore, material flows in and out of tribological contacts cannot be fully understood, especially under changing physicochemical conditions.

The third body concept is a promising model for lubrication in various environments. ${ }^{[10-15]}$ It suggests that a thin layer, called the third body, is progressively formed inside the contact to accommodate velocities and transfer loads between the bodies initially in contact, the first bodies. Although experimental observation has demonstrated the existence of solid and gaseous flows within the contact, ${ }^{[6-9,16-18]}$ observation inside contacts between engineering materials such as steel remains challenging. Numerical modeling based on discontinuous mechanics, for example Molecular Dynamics (MD) at the nano-scale ${ }^{[19,20]}$ and Discrete Element Method (DEM) at the micro-scale, ${ }^{[20-23]}$ are promising frameworks for artificially looking inside a contact of interest to understand and predict the tribological behavior of real lubricated mechanisms. Although MD simulations can efficiently account for physicochemical reactions, ${ }^{[19]}$ both the time and geometrical scales of DEM simulations are relevant to macroscale applications. However, the interactions between elements must be defined and measured carefully to simulate the expected behavior and not an artificial one.

The objective of the present work is to characterize those interactions by measuring the work of adhesion (W) in dry lubricated contacts to ultimately inform a DEM model with standard data. The ultimate goal is to predict the tribological behavior of materials using this model. The study focuses on $\mathrm{MoS}_{2}$-lubricated AISI 440C stainless steel contacts from both a laboratory scale configuration (pin-on-plate reciprocating tribological testing in ultrahigh vacuum) and from ball bearings mounted on an engineering model of a spacecraft instrument. The laboratory-tested samples were used to study the variation of $W$ between those engineering materials and the third bodies created at different key stages of their friction lives in comparison with measurements done on the ball bearings. The present work is unique in that the materials in the contacts under study are real engineering materials, as opposed to materials that have or can easily achieve ideal geometries. These 
macroscopic samples can be "chaotic" (i.e., high roughness and third body particles that can be easily displaced even during routine Atomic Force Microscopy (AFM)), presenting both experimental and theoretical challenges, as most adhesion models only apply to surfaces that are either flat ${ }^{[24]}$ or have nanoscale roughness. ${ }^{[25-30]}$

Measuring $W$ requires measuring the pull-off or adhesion force between the materials under consideration. However, this is a situational measure of adhesion, since it does not account for the geometry of the contact and is therefore not an intrinsic property of the contact. Moreover, adhesion force has been shown to be sensitive to the mechanical and physicochemical properties of the surfaces in contact, such as Young's modulus and free energy of oxide formation. ${ }^{[31]}$ To account for the geometry, Sahoo et al. ${ }^{[32]}$ measured the adhesion of $\mathrm{MoS}_{2}$ microand nanoparticles and normalized their adhesion forces by dividing them by the radius of the AFM tip. Such normalization gives a similar solution to what would be obtained by considering a sphere-on-flat contact configuration, such as in the Derjaguin-Muller-Toporov (DMT) and Johnson-KendallRoberts (JKR) models. ${ }^{[24]}$ In both models, the pull-off force only depends on the $W$ and the sphere radii. Therefore, a limitation of the JKR and DMT models is that they do not describe adhesion between rough surfaces. However, they can be employed to study adhesion of nominally flat rough surfaces using a statistical approach ${ }^{[33,34]}$ and by taking into account the contribution of each local asperity-surface contact in the global force of adhesion. ${ }^{[33]}$ Fairly reliable at the microscale, different models such as the Rumpf, ${ }^{[26,27,35,36]}$ modified Rumpf, ${ }^{[28,29]}$ and Katainen ${ }^{[27,30]}$ models might be more relevant when studying nanoscale roughness. Those three models allow for a "true" $W$ that is, a value of $W$ closer to the thermodynamic $W$ rather than a "practical" one as given by JKR or DMT. ${ }^{[37]}$ Experimental studies notably showed that the "true" $W$ can be 2 to 25 times the "practical" one. ${ }^{[28,38]}$

Finally, humidity is also of interest in adhesion studies since it can induce capillary forces, which can have a significant effect on experimentally measured adhesion forces. ${ }^{[38-40]}$ Considering the sheer number of factors that affect adhesion force as well as its

different components, there is a need for $W$ as a normalized, comparable measures of adhesion between surfaces. The present work will outline a protocol for measuring the $W$ of contacts that are more realistic when compared to real applications than those investigated in many previous adhesion studies.

\section{Experiments and Methodology}

An Asylum Research MFP-3D AFM was used for all imaging and adhesion measurements. NanoWorld AG NCHR sharp tip Si cantilevers were used for high-resolution imaging of regions of interest. Tipless NANOSENSORS ${ }^{\mathrm{TM}}$ TL-NCH cantilevers modified in-house with $\approx 10 \mu \mathrm{m}$ AISI $440 \mathrm{C}$ stainless steel beads (Sandvik Osprey Ltd) were used for adhesion measurements. The AISI 440C beads were cleaned of contaminants using a Bransonic ${ }^{\circledR} \mathrm{M} 1800 \mathrm{H}$ ultrasonic cleaner in acetone and ethanol for 5 min each, and then dried in an oven (Cole-Parmer ${ }^{\circledR}$ Model 281A) for $30 \mathrm{~min}$ at $45 \pm 5^{\circ} \mathrm{C}$. They were glued to the cantilevers using PC-SuperEpoxy. To remove any contamination covering the interacting surface of the bead, the cantilevers were submerged in acetone, then ethanol to wash away the acetone and any other residues for $1 \mathrm{~min}$. Figure 1 shows SEM images and EDX spectra of a clean-beaded cantilever. The interacting surfaces of the beads were imaged using a different, sharp-tip AFM cantilever before and after adhesion measurements to characterize their shape and any particle contamination.

Adhesion measurements were performed on three samples that underwent macroscale tribological tests. Each sample represents a different stage in the wear life of $1 \mu \mathrm{m}$ thick $\mathrm{MoS}_{2}$ coating $^{[16,41]}$ (Figure 1): early stages of friction (3 cycles of friction), transient stages ( 10 cycles of friction), and steady state (150 cycles of friction). These samples allow study of the evolution of the $W$ at the different key stages of the contact life. The samples used for this study are the same as those used in an earlier Time-of-Flight Secondary Ion Mass Spectroscopy (ToFSIMS) study by Colas et al., so the chemical compositions of the samples are well-understood. ${ }^{[41]}$ They were stored in a dry $\mathrm{N}_{2}$ environment to limit contamination, namely, physisorption of $\mathrm{N}_{2}$ replacing $\mathrm{H}_{2} \mathrm{O}$ at oxidation sites on $\mathrm{MoS}_{2}$ and slow formation of thin oxide layers under low humidity. ${ }^{[31,42,43]}$ The samples have a friction track, mostly composed of first body film (plasticized coating at top surface) and third body layer (an independent body created under friction), as well as untested, "pristine" coating outside the friction track. The friction tests were done under pure sliding over $\pm 15 \mathrm{~mm}$ reciprocating (with one complete reciprocation constituting one cycle of friction) at room temperature and ultrahigh vacuum $\left(10^{-6} \mathrm{~Pa}\right)$. The maximum Hertzian contact pressure was $1 \mathrm{GPa}$. It was calculated using a Hertz model considering a paraboloid (barrel shaped pin) on flat (coated substrate) configuration. Due to the size of the samples and the normal load $(1000 \mathrm{~N})$, the coating's properties are not taken into account, only the stainless steel's. The sliding velocity was $10 \mathrm{~mm} \mathrm{~s}^{-1}$.

Two $50 \times 50 \mu \mathrm{m}$ regions of interest (ROIs) were chosen inside and outside the friction track on each sample and imaged using a sharp tip AFM cantilever. Within each ROI, $10 \times 10 \mu \mathrm{m}$ sub-regions were then imaged with high resolution; three if inside the friction track and two if outside. The former was more heterogeneous than the latter and thus benefitted from additional measurements. These sub-regions were chosen carefully to be as flat and homogeneous (in terms of morphology and local features) as possible because adhesion was measured at three different points within each sub-region. The measurements were performed with a dwell time of $1 \mathrm{~s}$ (i.e., contact was maintained for $1 \mathrm{~s}$ before unloading) and using the $50 \times 50 \mu \mathrm{m}$ map of the ROI reimaged using the beaded cantilever. At each location, five applied loads were tested, starting at $2 \mu \mathrm{N}$ and increasing by 2 up to $6 \mu \mathrm{N}$, then decreasing by the same step back to $2 \mu \mathrm{N}$. Each load was applied six times in a row to obtain six adhesion force values, reducing random error by averaging and testing local reproducibility of the measurements.

As the study aims to provide insight into adhesion at three different stages in the tribological life of an $\mathrm{MoS}_{2}$-based lubricant, the pristine coating outside the friction track serves as a control between all three samples. All three were deposited in the same batch and should exhibit the same properties.

The tests were done in ambient air at approximately $25 \pm$ $2.5 \%$ and $55 \pm 5 \%$ relative humidity $(\mathrm{RH})$. The AFM was 
Tribological tests in UHV $\left(10^{-6} \mathrm{~Pa}\right)$

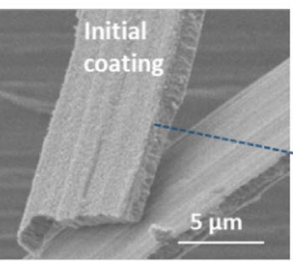

\section{$\underline{3 \text { cycles }}$}
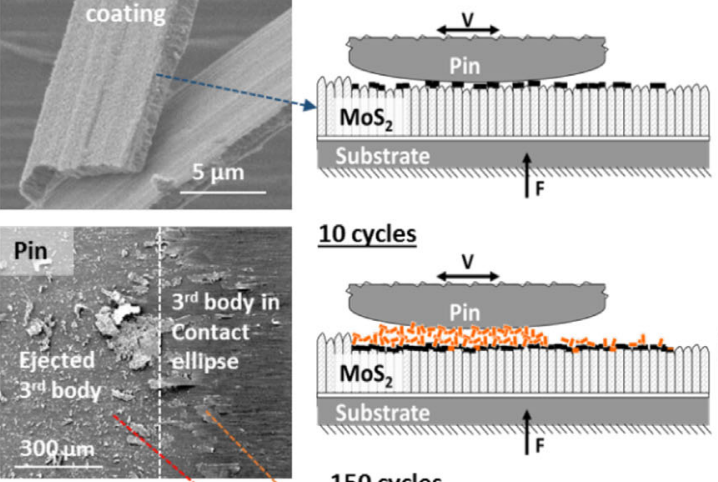

10 cycles

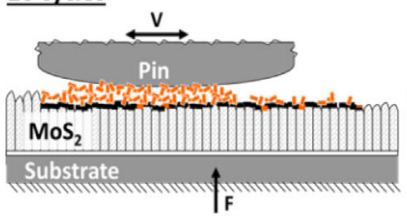

150 cycles

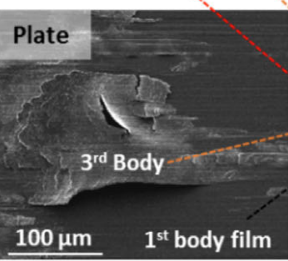

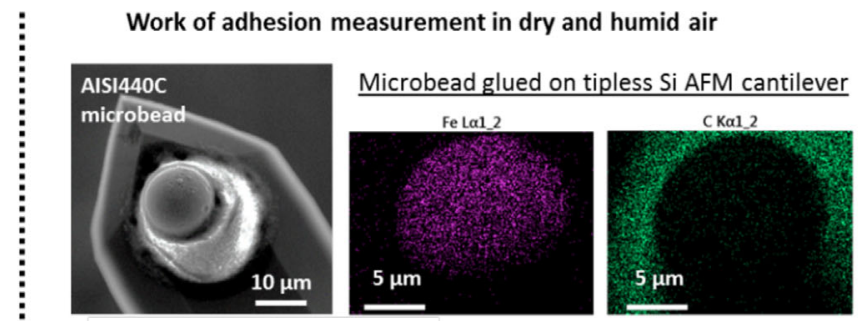

$\vdots$

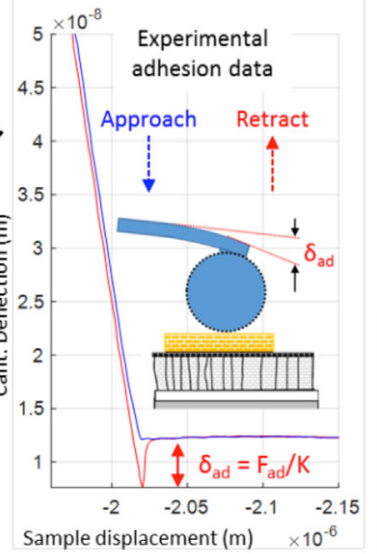

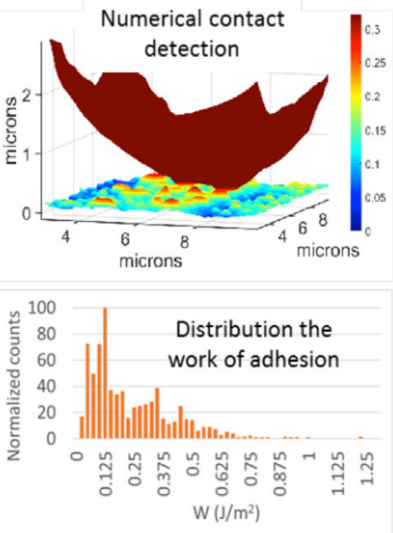

Figure 1. (Left) Summary of the study from the tribological tests conducted to the measurement of $W$. On the left, schematics and SEM images of the contact and surfaces at the different stages of friction (running-in, transient, and steady state). (Right) SEM image of the AISI 440C steel microbead of a clean-beaded AFM cantilever and EDX analysis. The principle of adhesion force measurement is also schematized along with the detection of contact asperities done numerically (using AFM high resolution images of the surfaces) to ensure reliable calculation of the work of adhesion. $K$ is the cantilever stiffness; $F_{a d}$ is the adhesion force measured.

equipped with a humidity sensor inside the acoustic chamber to continuously monitor humidity. Moreover, for each sample, a different cantilever was used for measurements inside and outside the friction track to limit the effects of contamination and material transfer. A total of twelve cantilevers were used: two per each of the three samples at the two different $\mathrm{RH}$ values.

The adhesion force $F_{a d}$ is calculated using Hooke's law (Figure 1). To obtain a more normalized measure of adhesion, the $W$ was calculated using the DMT contact model. This model was chosen because the materials are stiff and the surface roughness is primarily larger than the nanometer scale. Moreover, the materials (coating, first and third bodies) exhibit a complex chemical $\mathrm{Mo}_{\mathrm{x}} \mathrm{S}_{\mathrm{y}} \mathrm{O}_{\mathrm{z}}$ nature which does not, at this point, allow reliable determination of the range of adhesion $z_{0}$ used in some nanoscale adhesion models. ${ }^{[26-30]}$ Assuming contact between a sphere with a calculated global bead radius (least-squares sphere fit of the whole bead) and a flat, $W$ values were shown to be fairly unrealistic (equal to only a few $\mathrm{mJ} \mathrm{m}^{-2}$ ). A further study of the surfaces showed that the radii of curvature of asperities are not small compared to the local radius of the bead.

A MATLAB ${ }^{\circledR}$ script was developed to detect interacting asperities, their radii, and the semi-local bead radii by emulating the geometrical interpenetration of the bead in the surface (see SI 1 for details). This is an important step as the real contact may happen at a different location than the targeted one (Figure 2), more particularly because the shape of the bead is not perfectly spherical. The radii of the asperity and bead were then used to calculate the reduced radius $R^{*}\left(1 / R^{*}=1 / r+1 / R\right)$ of an equivalent sphere-on-sphere contact according to the Derjaguin approximation, ${ }^{[44,45]}$ which in turn is used to calculate $W$. In cases of multi-asperity contacts, the sum of the reduced radii may be taken. If the total measured pull-off force $F_{a d, T}$ is the sum of the pull-off forces at each asperity contact, then $W$ may be calculated using Equation 1.

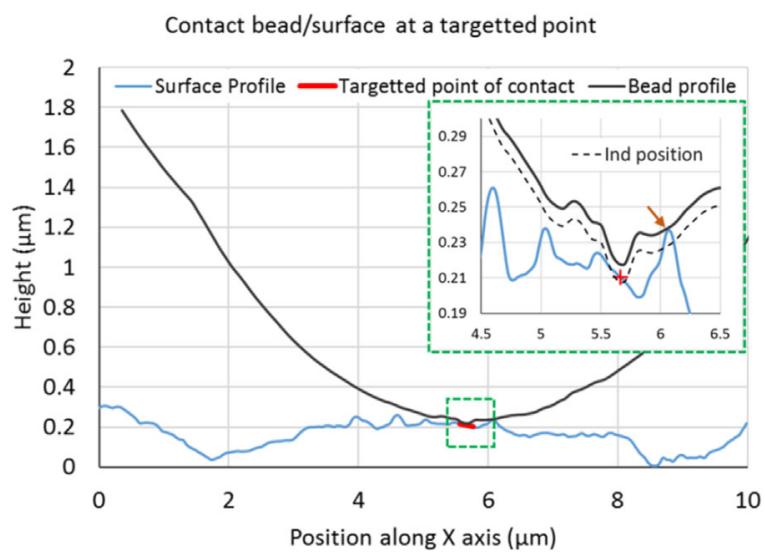

Figure 2. Extracted line profile from AFM scan showing the real asperity coming into contact (highlighted by the brown arrow) with the targeted point (red cross in the inset). This is the flattest region found using optical microscope and AFM after friction tests on the lab sample. Note that in this particular case, the targeted point comes into contact with the bead at maximum interpenetration depth shown by the "ind. position" dotted line in the inset. 


$$
\mathrm{W}=\frac{F_{a d, T}}{2 \pi \sum_{i=1}^{n} R_{i}^{*}}
$$

The geometrical interpenetration emulation does not account for deformation due to normal loading, as only the radii of contacting asperities are needed to calculate W. Moreover, the measurements were intentionally conducted in the elastic regime. Other assumptions in the experiment included no effects due to capillary action and electrostatic forces. To properly evaluate the asperity radii and the $W$, numerical errors and AFM positioning errors were taken into account (see the SI 2 for detailed information).

\section{Results and Discussion}

\subsection{Adhesion Forces}

The raw force-distance curves measured by the AFM showed no evidence of energy dissipation characteristic of plastic deformation, suggesting that all measurements were within the elastic regime. However, at every location, as the loading (indentation force) initially increases, the adhesion force also increases, which contradicts the DMT model. Moreover, the rate of increase differs from location to location. Then, as the indentation force subsequently decreases, the adhesion forces tend to stabilize, or decrease at a significantly slower rate (see SI 2). Interestingly, AFM high resolution images of the surfaces after adhesion measurements showed no detectable modification of the surfaces. Therefore, only speculations can be drawn on the origin of such hysteresis, as discussed in SI 2.

In the following, only the stabilized values of adhesion force are used in the calculation of the average adhesion force, and subsequently in the calculation of $W$. The stabilization, which is observable at each indentation, is a reproducible characteristic of the experiment. Hence, it allows reliable comparisons between the different ROI and samples.

\subsection{Work of Adhesion}

Figure 3 shows the distributions of $W$ values obtained at $25 \pm 2.5 \%$ $\mathrm{RH}$ for measurement both inside and outside the friction tracks for all laboratory samples. In general, the distributions demonstrate that the properties outside of the friction tracks (the original coatings) are similar, as most values are within the $0.05-0.45 \mathrm{~J} \mathrm{~m}^{-2}$ range. Regarding the inside, the distributions exhibit similarities between one another but are very different from the outside distributions. Most values of $W$ are within the $0.05-0.6 \mathrm{~J} \mathrm{~m}^{-2}$ range. In both cases, the range of measured values of $W$ is relevant to values reported in the literature with different pairs of materials. ${ }^{[38,46-48]}$

More specifically, in the outside region of the friction track, the longer tail observed in the 10 cycle sample distribution appears to come from the contamination of the bead by $\mathrm{MoS}_{2}$ particles (see SI 3 for details). Indeed, in that particular case, the coating was more prone to release particles during the adhesion measurements and locally covered the bead with them. A non-negligible contribution of $\mathrm{MoS}_{2}-\mathrm{MoS}_{2}$ adhesion force might explain the wider range of possible values for $W$ and the longer tail of the distribution toward high values. Consequently, considering all outside of the friction track data, we can assume that the work of adhesion between AISI440C steel and the $\mathrm{MoS}_{2}$ coating studied here can be estimated to be $W_{\text {AISI } 440 C / M o S 2} \approx 225 \mathrm{~mJ} \mathrm{~m}^{-2}$. Compared to the inside of the friction track of all three samples, it appears that friction tends to generally decrease $W$ toward values ranging from 0.05 to $0.2 \mathrm{~J} \mathrm{~m}^{-2}$, identified with the red dotted lines on the histograms. It is interesting to note that the 3 and 150 cycles samples exhibit similar shapes of distribution, with three different distinguishable regions. Compared to the 3 cycle sample, after 150 cycles the main red peak is more dominant than the other two. The 10 cycle sample also exhibits all three peaks, however the second peak is less distinguishable than with the other two samples. Finally, the distribution from the 10 cycle sample shows a longer tail.

The above similarities and differences are consistent with the friction life of the contact in terms of creation, morphology, and chemical compositions of the first and third bodies inside the contact. ${ }^{[16,41]}$ Indeed, at 3 cycles of friction (Figure 1), the surface of the coating is mainly plasticized on top (over a few tens of nanometers only) with only marginal particle detachment. Consequently, the contact is mainly constituted of the first body film. At 10 cycles of friction (Figure 1), significant particle detachment occurs; that is, the creation of the third body material is activated. These particles are composed of plasticized patches that have newly detached (formerly first body film), leaving underneath a "pristine" coating exposed to the environment and marginally thicker elements comprising both the coating and the first body film. Consequently, the contact is comprised of first body film, freshly exposed pristine coating, and third body particles. Finally, at 150 cycles (Figure 1), the contact is at steady state and comprised of a stable third body layer, that is, an agglomerate of third body particles. Those particles have been compacted together and continuously stressed together inside the contact during sliding. Chemical studies of the contact based on conventional mass spectrometry, EDX, and Tof-SIMS analysis show that during friction, an evolutionary chemical rearrangement occurs. ${ }^{[16,41]}$ It was shown that friction induces desorption of contaminants mostly composed of carbonaceous species $\left(\mathrm{CO}, \mathrm{CO}_{2}, \mathrm{C}_{\mathrm{x}} \mathrm{H}_{\mathrm{y}}\right), \mathrm{H}_{2} \mathrm{O}$ and $\mathrm{H}$. The ToF-SIMS study also showed that the initial coating is not pure $\mathrm{MoS}_{2}$ and is turned into a $\mathrm{MoS}_{\mathrm{x}} \mathrm{O}_{\mathrm{y}}$ first body film and third body layer under friction. Table 1 shows that the chemical composition of the original coating (outside friction track) is the same on all samples, confirming the homogeneity that was expected as they are all done in the same batch. Regarding the inside of the friction track, the 3 cycle sample and 150 cycle sample exhibit very similar composition, with 150 cycle sample more different from the outside than the 3 cycle sample is. The 10 cycle sample has a chemical composition that is a mix of both the outside and the inside of the friction track.

Regarding the contact angle (CA) measurements, the literature ${ }^{[49]}$ shows that the addition of oxygen into $\mathrm{MoS}_{2}$ makes the surfaces more hydrophilic because oxygen has a higher capability to form hydrogen bonds with water than the Sulfur atoms. Moreover, if $\mathrm{MoS}_{2}$ surfaces are contaminated with 


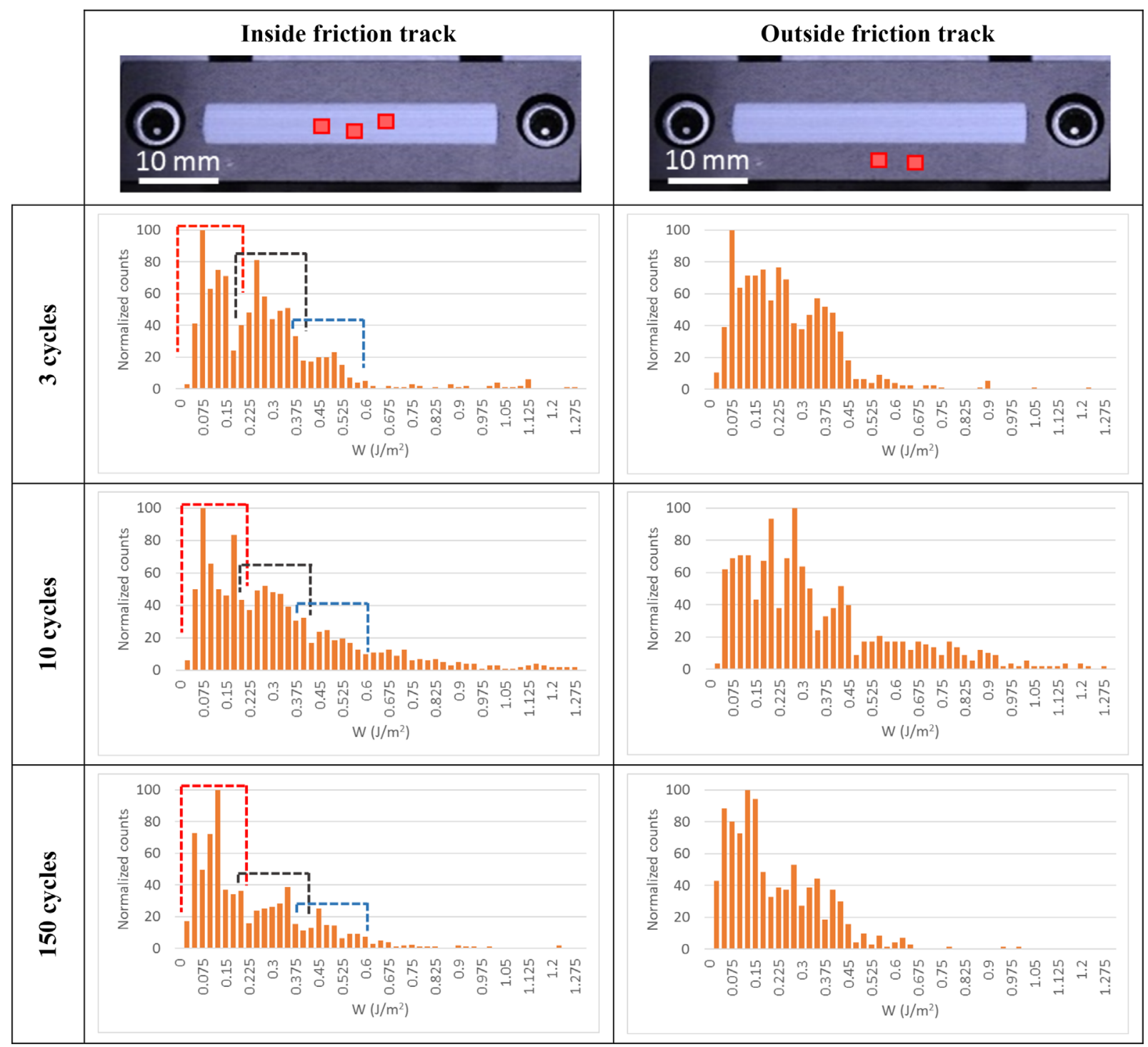

Figure 3. Distributions of calculated works of adhesion $W\left(\mathrm{~m}^{-2}\right)$ from the adhesion measurements performed in air $25 \pm 2.5 \%$ inside and outside the friction tracks.

Table 1. Relative intensity of the main ions related to the chemical composition of the inside and outside of the friction track of the different samples, measured by ToF-SIMS analysis (values reproduced from ref.[41]), and contact angle of water droplets on the different surfaces.

\begin{tabular}{|c|c|c|c|c|c|c|c|}
\hline \multirow[b]{2}{*}{ ToF-SIMS Ion } & & \multicolumn{2}{|c|}{3 Cycles } & \multicolumn{2}{|c|}{10 Cycles } & \multicolumn{2}{|c|}{150 Cycles } \\
\hline & & Outside & Inside & Outside & Inside & Outside & Inside \\
\hline $\mathrm{MoS}_{2}^{-}$ & & $23.6 \pm 0.6$ & $6.6 \pm 0.8$ & $23.4 \pm 1.2$ & $20.2 \pm 1.4$ & $27.1 \pm 3.0$ & $9.9 \pm 0.8$ \\
\hline $\mathrm{MoSO}^{-}$ & & $24.2 \pm 0.6$ & $5.9 \pm 0.7$ & $24.9 \pm 0.2$ & $7.2 \pm 0.3$ & $26.5 \pm 2.1$ & $9.6 \pm 0.6$ \\
\hline $\mathrm{MoS}_{2} \mathrm{O}^{-}$ & & $12.8 \pm 0.4$ & $4.6 \pm 0.6$ & $12.9 \pm 0.8$ & $7.6 \pm 0.5$ & $15.3 \pm 1.7$ & $7.3 \pm 0.6$ \\
\hline $\mathrm{MoS}_{3}^{-}$ & & $8.3 \pm 0.2$ & $4.1 \pm 0.5$ & $8.3 \pm 0.5$ & $9.3 \pm 0.7$ & $10.0 \pm 1.0$ & $7.1 \pm 0.6$ \\
\hline $\mathrm{Mo}_{2} \mathrm{~S}_{3}^{-}$ & & $25.1 \pm 0.5$ & $2.6 \pm 0.7$ & $25.0 \pm 1.3$ & $9.0 \pm 0.1$ & $27.8 \pm 3.0$ & $4.2 \pm 0.4$ \\
\hline Contact Angle $\left({ }^{\circ}\right)$ & $\mathrm{MoS}_{2}$ & $74 \pm 1.3^{a)}$ & $61.3 \pm 0.9$ & $74 \pm 1.3^{\mathrm{a})}$ & $56 \pm 2.2$ & $74 \pm 1.3^{\mathrm{a})}$ & $60.1 \pm 1.1$ \\
\hline & AISI $440 \mathrm{C}$ & & & & & & \\
\hline
\end{tabular}

a) The measurements were done on a Si wafer coated with the same $\mathrm{MoS}_{2}$ coating. The outside of the friction track was too narrow for a droplet of sufficient size to measure contact angle and measurements were influenced by the friction tracks. 
carbonaceous species, it becomes more hydrophobic than pristine $\mathrm{MoS}_{2}$, exhibiting a $\mathrm{CA} \approx 90^{\circ[49,50]}$ compared to $\mathrm{CA} \approx$ $70^{\circ[49]}$ initially. The hydrophobicity is impeded if the $\mathrm{MoS}_{2}$ is slightly defective $\left(\mathrm{CA} \approx 70^{\circ}\right)^{[50]}$ or if the sample is made of microsized particles $\left(\mathrm{CA} \approx 75^{\circ}\right){ }^{[51]}$ These observations are consistent with the differences in CA measured inside the friction track after 3 and 150 cycles of friction $\left(\mathrm{CA} \approx 60^{\circ}\right)$ and outside the friction track $\left(\mathrm{CA} \approx 74^{\circ}\right.$ ) (Table 1$)$. Indeed, the inside is shown to be comprised of a $\mathrm{MoS}_{\mathrm{x}} \mathrm{O}_{\mathrm{y}}$ material, while the outside shows higher detection of $\mathrm{Mo}_{2} \mathrm{~S}_{3}{ }^{-}$molecular ions and lower detection of $\mathrm{MoS}_{\mathrm{x}} \mathrm{O}_{\mathrm{y}}{ }^{-}$molecular ions (Table 1). Additionally, the coating is made of nano-sized crystallites mostly oriented perpendicular to the substrate ${ }^{[52,53]}$ which is a hydrophilic orientation $(\mathrm{CA} \approx$ $\left.40^{\circ}\right) \cdot{ }^{[50]}$ That coupled with the inherent carbonaceous contamination of the pristine coating ${ }^{[41]}$ makes the CA measured outside the contact highly relevant to the nature of the surface. The 10 cycle sample shows the highest hydrophilicity $\left(\mathrm{CA} \approx 56^{\circ}\right)$ although it exhibits a very high detection of $\mathrm{Mo}_{\mathrm{x}} \mathrm{S}_{\mathrm{y}}{ }^{-}$molecular ions. At 10 cycles of friction, regions of pristine coating are exposed to the environment due to particle detachment. Consequently, edges of $\mathrm{MoS}_{2}$ crystallites are exposed to the environment. Moreover, the coating was shown to be cleaned of contaminants during friction. ${ }^{[16,41]}$ That makes the measured CA consistent with the morphological and chemical nature of the surface in a sense that the exposed coating significantly contributes to the decrease of the CA.

The CA and $W$ are both linked to the surface energies of the surfaces. ${ }^{[49-51,54]}$ In general, when the CA is low, W should be high. This is consistent with what is observed inside the friction track as higher $W$ is measured on the 10 cycle sample compared to the 3 and 150 cycles sample. However, $W$ outside the friction track is the highest when it should be the lowest based on the CA. The literature shows that some discrepancies can occur between the calculated $W$ using surface energies and the $W$ measured using contact mechanics models. ${ }^{[54]}$ The case of outside the friction track might have a geometrical configuration under loading that could accentuate interactions with the steel. Based on surface energy, $\mathrm{MoS}_{2}$ is known to have higher adhesion to

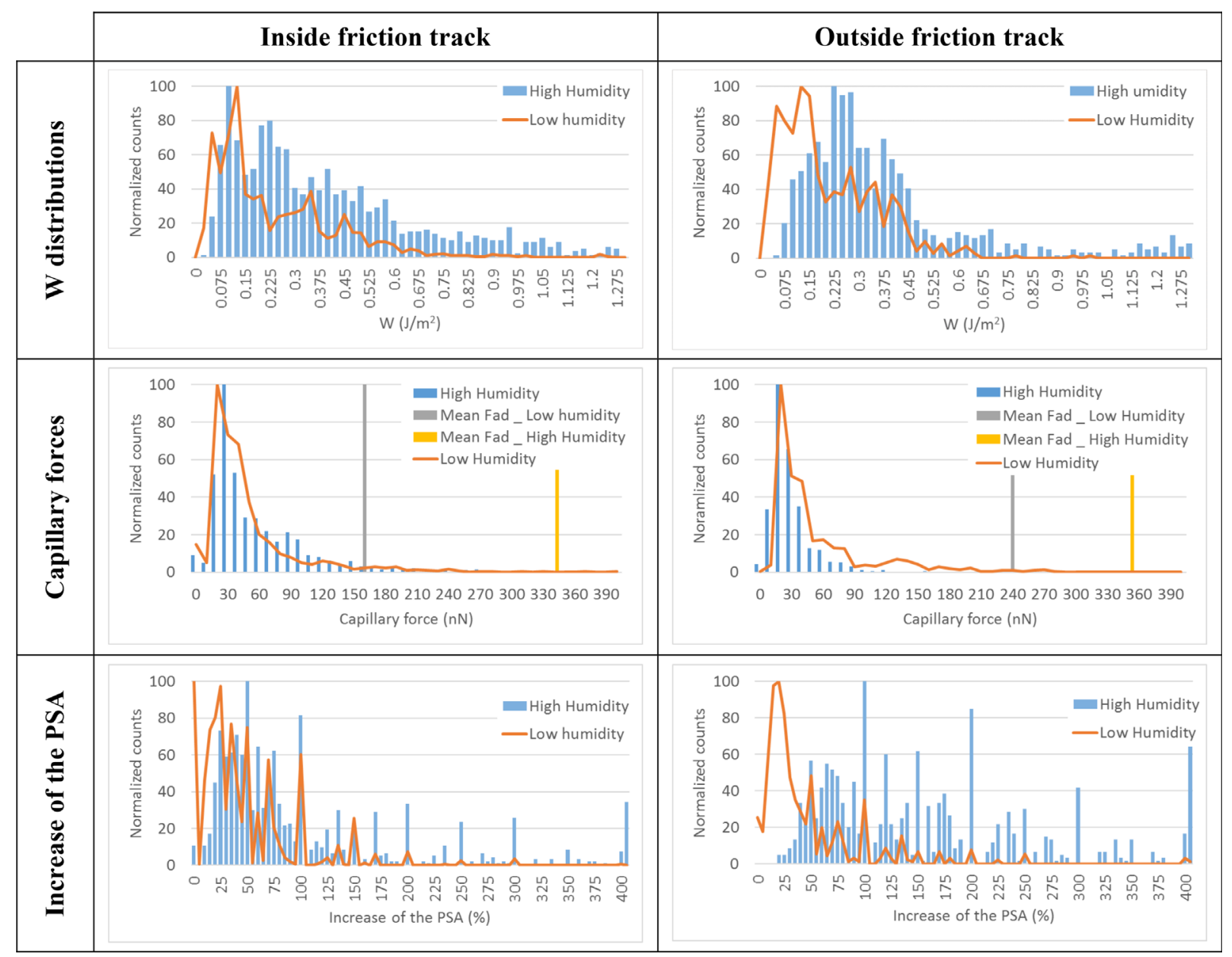

Figure 4. Effect of humidity on the determination of the work of adhesion inside and outside the friction track of the 150 cycle sample. The figure shows the distribution of $W$ at $58 \%$ (inside) and $60 \%$ (outside) of relative humidity. The second row shows the distribution of the capillary forces calculated from the Derjaguin approximation, with indications of the mean adhesion force $F_{a d}$ measured at low and high humidity in both dry and humid environments. The third row displays the effect of humidity on increasing the projected surface area when considering the regions of the contact where a capillary bridge can form. 
steel when it is in contact on its edges versus on the plane. ${ }^{[55]}$ In our experiments, the loading might remove the weakly absorbed carbonaceous contaminants from the surface leaving the perpendicularly oriented crystallite in contact with the steel microbead and inducing high adhesion (that is, high W).

Consequently, at $25 \pm 2.5 \% \mathrm{RH}$, the distributions of $W$ shown in Figure 3 are consistent with the composition of the friction track. Interestingly, the 10 cycle sample exhibits a distribution, which seems to be a combination of the inside and outside of the friction track. Indeed, the main peak and the lowest peak, respectively, highlighted with the red and blue dotted lines are related to the inside while the second peak of the distribution, which is less distinguishable, is related to the outside.

At $55 \pm 5 \% \mathrm{RH}$, there is less consistency in the distributions of $W$ values measured. In high humidity, the capillary forces may have a significant contribution to the measured forces. ${ }^{[56]}$ Considering the surfaces studied here, capillary forces are difficult to reliably evaluate, particularly if capillary bridges are forming at the contact. ${ }^{[57]}$ To qualitatively study the effect of the capillary forces, we first used the Derjaguin approximation assuming a liquid bridge is forming at the contact. However, the approximation is valid only when contact is made between

the bead and the asperity; that is, no capillary bridge is formed at the very point of contact. ${ }^{[57]}$ Equation 2 represents the Derjaguin approximation in that case, where $\gamma$ is the surface tension of water $\left(\gamma=0.073 \mathrm{~J} \mathrm{~m}^{-2}\right), R^{*}$ is the reduced radius of the local radius of the bead and the radius of the asperity in contact, $\theta_{1}$ and $\theta_{2}$ are the contact angles of the surfaces.

$F_{c} \approx 2 \pi \gamma R^{*}\left[\cos \left(\theta_{1}\right)+\cos \left(\theta_{2}\right)\right]$

Secondly, we took into account the Kelvin radius $R_{k}$ (Equation 3), which allows the evaluation of the maximum separation distance $2 R_{k}$ between two surfaces to get a stable capillary bridge forming under the pressure and temperature conditions of the experiments. ${ }^{[56]}$ In Equation 3, $\gamma$ is the surface tension of water, $V_{m}$ is the liquid molar volume of water under the pressure and temperature conditions of the experiment, $R$ is the universal ideal gas constant, $T$ is the temperature in Kelvin, and $\frac{P}{P_{\text {sat }}}$ is the relative humidity. Based on the calculated maximum separation distance, the increase of the projected surface area (PSA) at $25 \pm 2.5 \% \mathrm{RH}$ and $55 \pm 5 \% \mathrm{RH}$ can be evaluated. It is then compared to a simple contact, where only solid interaction between the bead and the surface is considered. The PSA is calculated by counting the number of pixels of the surface coming into contact with the bead and the number of pixels of the surface impacted by capillary bridge formation The number of pixels is then multiplied by the area represented by one pixel.

$R_{k}=-\frac{\gamma V_{m}}{R T}\left[\ln \left(\frac{P}{P_{\text {sat }}}\right)\right]^{-1}$
Figure 4 shows a comparison of the results obtained for the 150 cycle samples at both $25 \pm 2.5 \% \mathrm{RH}$ and $55 \pm 5 \% \mathrm{RH}$. In terms of $W$ values, the contribution of values higher than $0.2 \mathrm{~J} \mathrm{~m}^{-2}$ is more dominant in humid air than in dry air. Moreover, the distribution exhibits a longer tail. Outside the friction track, the ranges of values in which the majority of $W$ values are (from 0.05 to $0.45 \mathrm{~J} \mathrm{~m}^{-2}$ ) are similar in humid air and dry air. However, the most prominent peak is significantly shifted by almost $0.2 \mathrm{~J} \mathrm{~m}^{-2}$. If the capillary force is calculated using Equation 2, no difference is observed between humid and dry air for both the inside and the outside of the friction track. However, if we consider the increase of the PSA as a function of the humidity, differences in $W$ distributions make sense. As shown on Figure 4, inside the friction track, the PSA increases by around $15 \%$ (main peak at $0 \%$ increase) in the dry air and by around $45 \%$ in humid air. Differences between humid and dry air are not significant, with most of the distributions overlapping. That may explain why the general shape of the $W$ distribution observed in dry air is still observable in humid air. Outside the friction track, the increase in the PSA is much higher in humid air (>70\%) compared to dry air (20\%). Multiple peaks are notably observed in the high range of values in humid

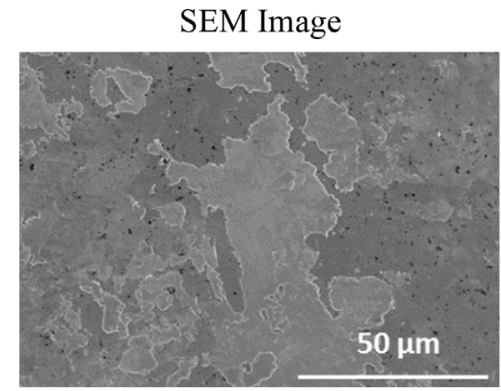

$\mathrm{S} K \alpha 1$

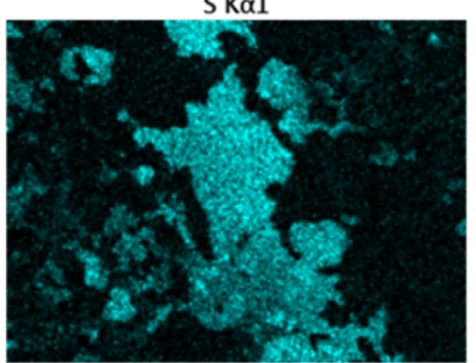

Mo L $\alpha 1$

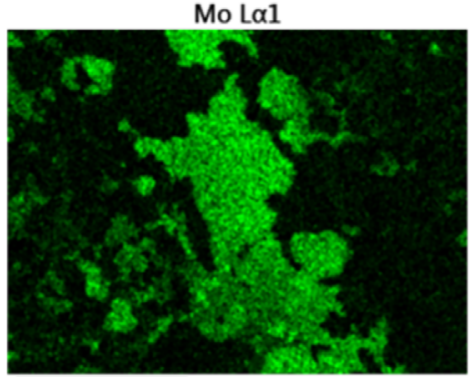

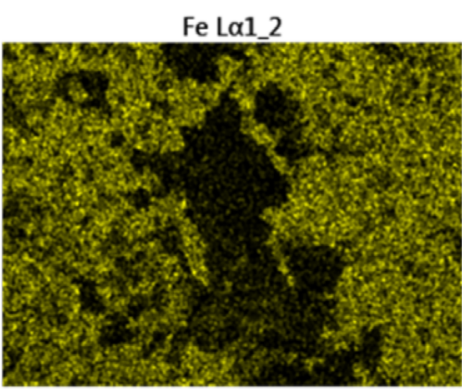

$\operatorname{Cr} L \alpha 12$

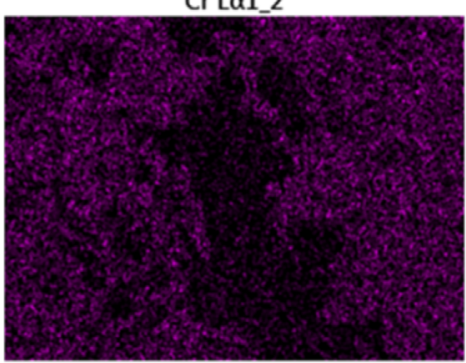

$\mathrm{O} K \alpha 1$

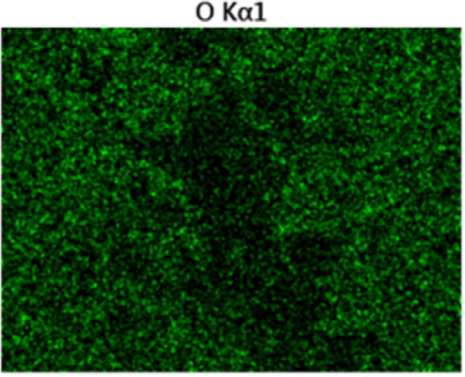

Figure 5. SEM image and corresponding elemental map of a typical flat, compacted third body layer observed on the ball of the EM-LTU bearing from the NIRISS DW mechanism. The maps were acquired with a beam energy of $10 \mathrm{keV}$. 
air. That large shift may explain the significant shift observed in the $W$ distribution obtained in humid air compared to the one obtained in dry air.

\subsection{Testing Protocol on a Real Contact}

To test the protocol with a contact from a real mechanism, measurements were done on a third body created in a dry lubricated ball bearing coming from the Engineering Model Life Test Unit (EM-LTU) of the Dual Wheel (DW) mechanism from the Near Infrared Imager and Slitless Spectrograph (NIRISS), an instrument to be used on the James Webb Telescope (JWST). ${ }^{[5]}$ The lubrication is ensured by a $\mathrm{MoS}_{2}$ coating is deposited on the races and the retainer, which is made of the self-lubricating composite material PGM-HT. The PGM-HT is made of glass fibers and $\mathrm{MoS}_{2}$ particles embedded in a PTFE matrix. Consequently, there are two possible sources of lubricant.

To determine the nature of the third body created inside the contact, prior to the adhesion measurements, the ball bearing underwent a complete investigation using optical microscope, SEM and EDX analysis. Interestingly, the lubrication is mainly due to the creation of a Mo $+\mathrm{S}+\mathrm{O}$ third body layer covering the races and the balls (Figure 5) and loose $\mathrm{MoS}_{2}$ particles that are trapped in the friction track of the ball on the retainer socket. The retainer does not appear worn in any way except for the loose $\mathrm{MoS}_{2}$ particles that, based on their morphology, come from the retainer. As the PTFE matrix and the fibers do not show any damage and as no fluorine is detected anywhere in the third body layer, it can be assumed that the third body layer is primarily $\mathrm{Mo}+\mathrm{S}+\mathrm{O}$-based materials.

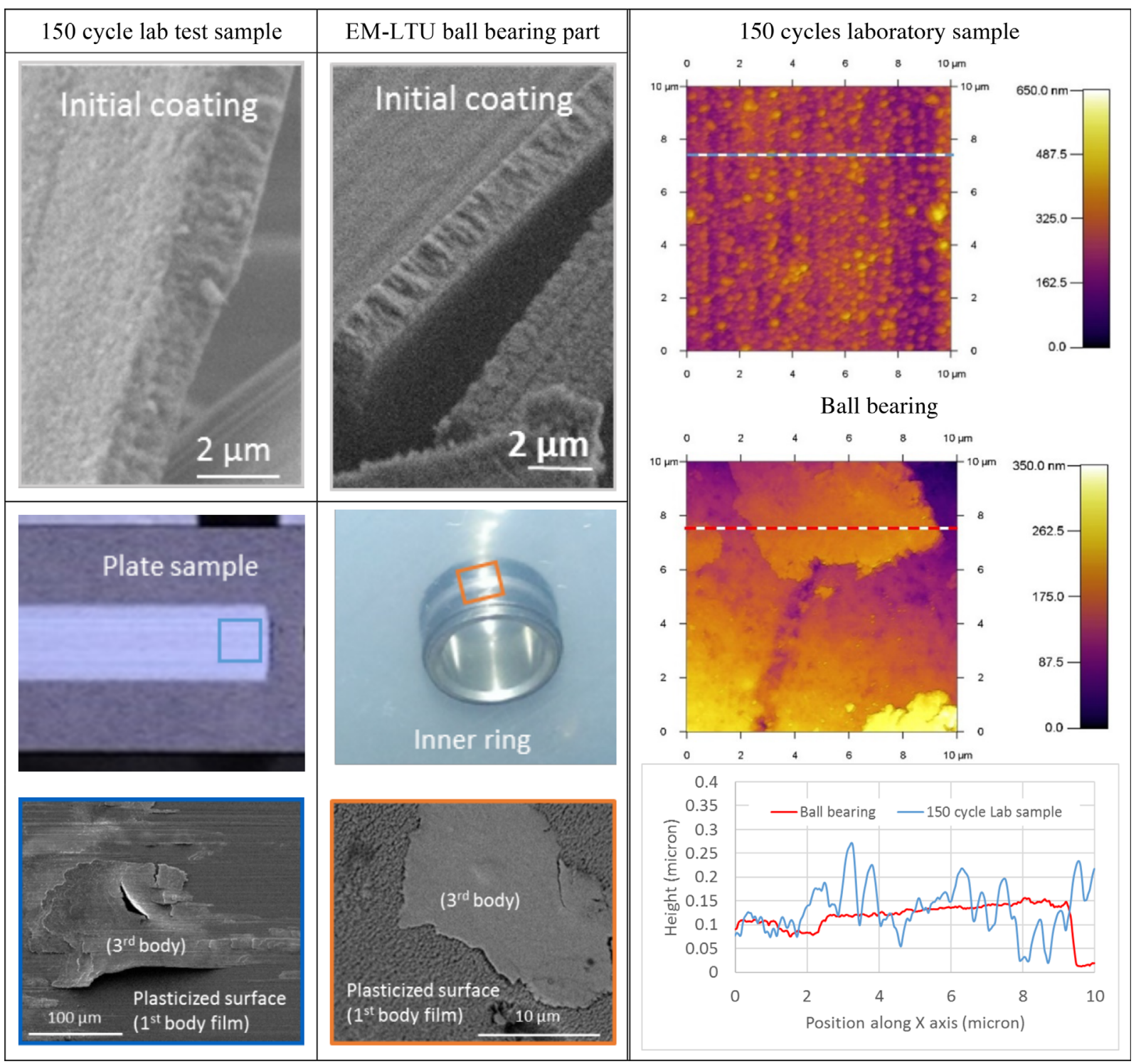

Figure 6. (Left) Comparison of the coatings, the third body, and the first body between the 150 cycle laboratory test sample and the EM-LTU ball bearing. (Right) Typical line profiles from the third body studied on the 150 cycle laboratory sample and the ball bearing. The targeted point of contact is at the x-position of $4.4 \mu \mathrm{m}$ on the laboratory sample and $6.1 \mu \mathrm{m}$ on the ball bearing. 


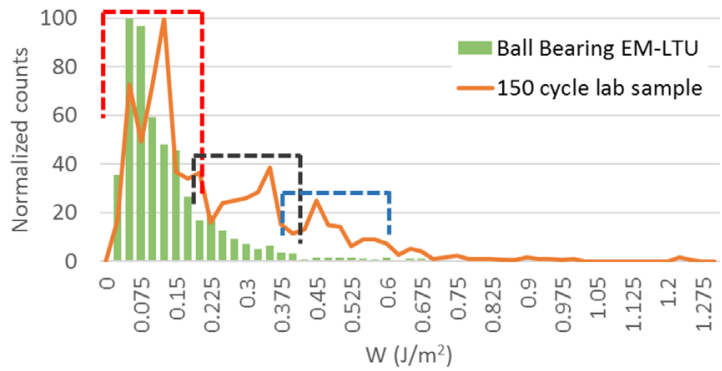

Figure 7. Distribution of the work of adhesion calculated on the third body created in a ball bearing initially mounted on the EM-LTU of the NIRISS Dual wheel mechanism of the JWST. Adhesion measurements were done in air, $22 \pm 1 \% \mathrm{RH}$. For comparison, the profile of the distribution obtained on the 150 cycle laboratory sample in dry conditions is also given.

It is very similar to the third body observed at steady state on the 150 cycle samples (both pins and plate) (Figure 6). The only significant difference is that it is extremely flat. This led to a slight change in the calculation of $W$ in order to be comparable with what was effectively done on the 150 cycle sample. In the ball bearing case, only the semi local radius of the bead was considered and a ball-on-flat configuration was used. This means that the reduced radius $R^{*}$ is identical to the semi local bead radius, which in turn means the model becomes equivalent to DMT.

Figure 7 shows the distribution of measured $W$ values over 25 different locations of interest. Adhesion measurements were performed in air $22 \pm 1 \% \mathrm{RH}$. In general, the distribution is much tighter than what was obtained on the laboratory sample. There is a good correlation with the distribution obtained with the 150 cycle sample in the sense that the main peak highlighted in red is detected in the $0.05-0.2 \mathrm{~J} \mathrm{~m}^{-2}$ range. A secondary peak is clearly detected. Both peaks are slightly shifted toward the low values compared to the peaks observed with the 150 cycle sample.

During the measurements, no issues of bead contamination with third body particles were encountered. Such contamination could have led to the addition of the contribution of third bodythird body contact to the measurement and to higher $\mathrm{W}$ values, as mentioned earlier with the 10 cycle sample (outside friction track, test in air $25 \pm 2.5 \% \mathrm{RH}$ ). The absence of bead contamination could easily explain why the distribution does not exhibit a long tail and possibly the absence of the third peak as detected with the 150 cycle sample. There are several other possible explanations for the differences observed. The reader is invited to refer to SI 4 for the related discussion. Nonetheless, based on those results and the discussion above, we can assume that the work of adhesion between AISI440C steel and the third body can be estimated to be $W_{\text {AISI I4AOC/3rd Body }} \approx 125 \mathrm{~mJ} \mathrm{~m}^{-2}$.

\section{Conclusion}

In the present work, a protocol has been developed to measure the work of adhesion (W) between $\mathrm{MoS}_{2}$ coatings and AISI 440C stainless steel before and after friction in ultrahigh vacuum. The protocol applied a Derjaguin approximation for computing W, which considered microscale roughness, to analyze AFM adhesion measurements of the $\mathrm{MoS}_{2} /$ steel interfaces. Measurements were conducted on both laboratory scale samples and macro-scale contacts in ball bearings. $W$ distributions measured in dry air revealed that throughout the friction life, $W$ is not constant and can vary along with the chemical nature of the materials. Inside the friction track, the calculated $W$ values were found to be correlated with (i) the material's chemical composition as revealed by ToF-SIMS and contact angle measurements, and (ii) with the orientation of $\mathrm{MoS}_{2}$ crystallites. $W$ on $\mathrm{MoS}_{\mathrm{x}} \mathrm{O}_{\mathrm{y}}$ materials was lower than on regions where crystallite edges are exposed, which is also consistent with CA measurements. Outside the friction track, $W$ is found to be the highest, such that $W$ for pristine $\mathrm{MoS}_{2}$ was found to be significantly higher than that of the third body created under friction $\left(W_{\text {AISI } 440 C / M o S 2} \approx 225 \mathrm{~mJ} \mathrm{~m}^{-2}\right.$ and $W_{\text {AISI } 440 C / 3 r d}$ Body $\approx 125 \mathrm{~mJ} \mathrm{~m}-2$ ). This indicates that friction in ultrahigh vacuum induced a decrease in adhesion energy between the steel and the lubricant due to tribochemical and structural modification to the contact surfaces. This is consistent with the reduction of friction observed with the studied samples. ${ }^{[41]}$ Measurements at high humidity revealed that the contribution of capillary forces can increase $W$ by more than $70 \%$. The distribution of $W$ values measured on the ball bearing showed a clear similarity with those conducted on the laboratory samples, and most importantly exhibited an equivalent $W_{\text {AISI440C/3rd Body }}$ This is a very promising outcome as conducting life tests on ball bearings is costly and using laboratory tests to get insight into the adhesion properties might reduce that cost. The works of adhesion measured here are currently being used in a DEM model for predicting friction and wear of $\mathrm{MoS}_{2}$-lubricated lubricated steel contacts currently under development.

\section{Supporting Information}

Supporting Information is available online from the Wiley Online Library or from the author.

\section{Acknowledgements}

S. P. and G. C. contributed equally to this work. We would like to thank the Department of Mechanical and Industrial Engineering at the University of Toronto for financial support (MIE Summer research award, summer 2015), the National Sciences and Engineering Research Council of Canada (Undergraduate Student Research Award, summer 2016, and Engage grant), Honeywell Aerospace (formerly COMDEV International Ltd) and more particularly Ashley McColgan, Peter Cameron, David Aldridge, and Alexander Beaton for their support and in-kind contribution to the project (EM-LTU ball bearings), the Canadian Space Agency and more particularly Andrew Gibson for his consultation support, the LaMCoS laboratory and the French Space Agency for their financial and technical support and allowing the use of laboratory samples coming from another dedicated study supported by CNES, the Canadian Foundation for Innovation (CFI) for financial support for equipment used in the work. We also thank Prof. Benjamin Hatton from the University of Toronto for assistance with contact angle measurements.

\section{Conflict of Interest}

The authors declare no conflict of interest. 


\section{Keywords}

work of adhesion, AFM, $\mathrm{MoS}_{2}$, roughness, tribology

[1] Y. Michel, E. Conde, D. Kuoach, M. Simpfendoerfer, Y. Parot, G. R. Orttner, M. Saccaccio, S. Maurice, in Proc. of the 13th European Space Mechanisms and Tribology Symposium, ESA SP-670, Vienna, Austria, ESA Communications, The Netherlands, 2009.

[2] M. Leckie, Z. Ahmad, in Proc. of the 40th Aerospace Mechanisms Symposium, NASA/CP-2010-216272, NASA Kennedy Space Center, Cocoa Beach, FL, United States, 2010, p. 401.

[3] A. S. Gibson, A. McColgan, J. C. Heald, M. Gentilhomme, B. Vila, Z. Dhanji, M. J. Anderson, R. Sharma, in Proc. of the 15th European Space Mechanisms and Tribology Symposium, ESA SP-718, Noordwijk, The Netherleands, ESA Communications, The Netherleands, 2013.

[4] A. S. Gibson, A. McColgan, J. C. Heald, E. Harpell, M. J. Anderson, R. Sharma, in Proc. of the 15th European Space Mechanisms and Tribology Symposium, ESA SP-718, Noordwijk, The Netherleands, ESA Communications, The Netherleands, 2013.

[5] D. Aldridge, M. Gentilhomme, A. Gibson, P. Cameron, A. Mccolgan, in Proc. of the 16th European Space Mechanisms and Tribology Symposium, ESA SP-737, Bilbao, Spain, ESA Communications, The Netherleands, 2015.

[6] A. Jullien, M. H. Meurisse, Y. Berthier, Wear 1996, 194, 116.

[7] S. Descartes, Y. Berthier, Wear 2002, 252, 546.

[8] K. J. Wahl, R. R. Chromik, G. Y. Lee, Wear 2008, 264, 731.

[9] B. Krick, J. Vail, B. J. Persson, W. G. Sawyer, Tribol. Lett. 2012, 45, 185.

[10] M. Godet, Wear 1990, 136, 29.

[11] M. Godet, Wear 1984, 100, 437.

[12] Y. Berthier, in Wear - Materials, Mechanisms and Practice (Ed: G. Stachowiak), John Wiley \& Son, Ltd, Chichester, West Sussex, England, 2005, p. 291.

[13] Y. Berthier, Wear 1990, 139, 77.

[14] G. Colas, Utilisation Raisonnée de Contaminants Pour Caractériser La Rhéologie Des 1ers et 3ème Corps Solides: Application À La Tribologie En Ambiances Spatiales, INSA de Lyon, 2013.

[15] M. Godet, Y. Berthier, J. Lancaster, L. Vincent, Wear 1991, 149, 325.

[16] G. Colas, A. Saulot, C. Godeau, Y. Michel, Y. Berthier, Wear 2013, 305, 192.

[17] G. Colas, A. Saulot, N. Bouscharain, C. Godeau, Y. Michel, Y. Berthier, Tribol. Int. 2013, 65, 177.

[18] G. Colas, A. Saulot, E. Regis, Y. Berthier, Wear 2015, 330-331, 448.

[19] J. Yeon, X. He, A. Martini, S. H. Kim, ACS Appl. Mater. Interfaces 2017, 9, 3142.

[20] M. Renouf, F. Massi, N. Fillot, A. Saulot, Tribol. Int. 2011, 44, 834.

[21] I. Iordanoff, A. Battentier, J. Néauport, J. L. Charles, Tribol. Int. 2008, 41, 957.

[22] M. Champagne, M. Renouf, Y. Berthier, J. Tribol. 2014, 136, 21603.

[23] M. D. Villavicencio, M. Renouf, A. Saulot, Y. Michel, Y. Mahéo, G. Colas, T. Filleter, Y. Berthier, Tribol. Int. 2017, 113, 362.

[24] K. L. Johnson, Tribol. Int. 1998, 31, 413.
[25] Y. I. Rabinovich, J. J. Adler, A. Ata, R. K. Singh, B. M. Moudgil, J. Colloid Interface Sci. 2000, 232, 10.

[26] O. Laitinen, K. Bauer, J. Niinimäki, U. A. Peuker, Powder Technol. 2013, 246, 545.

[27] S. N. Ramakrishna, L. Y. Clasohm, A. Rao, N. D. Spencer, Langmuir 2011, 27, 9972.

[28] T. D. B. Jacobs, K. E. Ryan, P. L. Keating, D. S. Grierson, J. A. Lefever, K. T. Turner, J. A. Harrison, R. W. Carpick, Tribol. Lett. 2013, $50,81$.

[29] B. Stegemann, H. Backhaus, H. Kloss, E. Santner, Mod. Res. Educ. Top. Microsc. 2007, 2, 820.

[30] J. Katainen, M. Paajanen, E. Ahtola, V. Pore, J. Lahtinen, J. Colloid Interface Sci. 2006, 304, 524.

[31] K. Miyoshi, in Solid Lubrication Fundamentals and Applications, CRC Press, New York, USA, 2001

[32] R. R. Sahoo, S. K. Biswas, Tribol. Lett. 2010, 37, 313.

[33] K. N. G. Fuller, D. Tabor, Proc. R. Soc. pf London A 1975, 345, 327.

[34] B. N. J. Persson, E. Tosatti, J. Chem. Phys. 2001, 115, 5597.

[35] Y. I. Rabinovich, J. J. Adler, A. Ata, R. K. Singh, B. M. Moudgil, J. Colloid Interface Sci. 2000, 232, 10.

[36] D. S. Grierson, E. Flater, R. W. Carpick, At. Force Microsc. Adhes. Stud. 2005, 3, 291.

[37] B. N. J. Persson, J. Chem. Phys. 2001, 115, 3840.

[38] T. Jiang, Y. Zhu, Nanoscale 2015, 7, 10760.

[39] T. Eastman, D. Zhu, Langmuir 1996, 12, 2859.

[40] D. L. Sedin, K. L. Rowlen, Anal. Chem. 2000, 72, 2183.

[41] G. Colas, A. Saulot, D. Philippon, Y. Berthier, D. Leonard, Thin Solid Films 2015, 588, 67.

[42] G. Colas, A. Saulot, Y. Michel, Y. Baudasse, A. Mistral, Y. Berthier, Proc. of the 15th European Space Mechanisms and Tribology Symposium, SA SP-718, Noordwijk, The Netherleands, ESA Communications, The Netherlands, 2013.

[43] M. N. Gardos, Tribol. Lett. 1995, 1, 67.

[44] B. Derjaguin, Kolloid-Zeitschrift 1934, 69, 155.

[45] S. Rentsch, R. Pericet-Camara, G. Papastavrou, M. Borkovec, Phys. Chem. Chem. Phys. 2006, 8, 2531.

[46] T. D. B. Jacobs, J. A. Lefever, R. W. Carpick, Adv. Mater. Interfaces 2015, 2, 1400547.

[47] E. S. Yoon, S. H. Yang, H. G. Han, H. Kong, Wear 2003, 254, 974.

[48] O. Dos Santos Ferreira, E. Gelinck, D. De Graaf, H. Fischer, Appl. Surf. Sci. 2010, 257, 48.

[49] P. K. Chow, E. Singh, B. C. Viana, J. Gao, J. Luo, Z. Lin, A. L. Elıas, Y. Shi, Z. Wang, M. Terrones, N. Koratkar, ACS Nano 2015, 9, 3023.

[50] A. P. S. Gaur, S. Sahoo, M. Ahmadi, S. P. Dash, M. J. Guinel, R. S. Katiyar, Nano Lett. 2014, 14, 4314.

[51] T. Kim, M. Amani, G. H. Ahn, Y. Song, A. Javey, S. Chung, ACS Nano 2016, 10, 2819.

[52] P. D. Fleischauer, M. R. Hilton, R. Baeur, Effects of Microstructure and Adhesion on Performance of Sputter-Deposited $\mathrm{MoS}_{2}$ Solid Lubricant Coatings, TOR-0090(5064)-2, 1990.

[53] J. Moser, J. Vac. Sci. Technol. A Vac. Surf. Film 1994, 12, 494.

[54] D. E. Packham, Int. J. Adhes. Adhes. 1996, 16, 121.

[55] A. I. Brudnyi, A. F. Karmadonov, Wear 1975, 33, 243.

[56] P. J. Van Zwol, G. Palasantzas, J. T. M. De Hosson, Phys. Rev. E - Stat. Nonlinear, Soft Matter Phys. 2008, 78, 1.

[57] Y. I. Rabinovich, M. S. Esayanur, B. M. Moudgil, Langmuir 2005, 21, 10992. 